\title{
RepC as a negative copy number regulator is involved in the maintenance of pJB01 homeostasis
}

\author{
Sam Woong Kim ${ }^{1}$, Ho Young Kang ${ }^{2}$, Sang Wan Gal ${ }^{3}$, Kwang-Keun $\mathrm{Cho}^{4}$ and Jeong Dong \\ Bahk $^{5 *}$ \\ ${ }^{1}$ Swine Science and Technology Center, Gyeongnam National University of Science and Technology, 150 Chilam-dong, \\ Jinju 660-758, Korea. \\ ${ }^{2}$ Department of Microbiology, College of Natural Sciences, Pusan National University, Busan 609-735, Korea. \\ ${ }^{3}$ Department of Pharmaceutical Engineering, Gyeongnam National University of Science and Technology, \\ 150 Chilam-dong, Jinju 660-758, Korea. \\ ${ }^{4}$ Department of Animal Resources Technology, Gyeongnam National University of Science and Technology, \\ 150 Chilam-dong, Jinju 660-758, Korea. \\ ${ }^{5}$ Division of Applied Life Sciences, Graduate School of Gyeongsang National University, Jinju 660-701, Korea.
}

Accepted 15 June, 2011

The plasmid pJB01 contains a single operon consisting of three orfs, $\operatorname{cop} A, \operatorname{rep} B$ and repC cistrons. The operon, also called repABC operon, starts transcription at T695 or A696 on the pJB01 genetic map. CopA (called RepA in pMV158 family) or ctRNA (counter-transcript RNA) of this plasmid play roles as a repressor of RepB, a replication initiator, on the transcriptional and translational level, respectively. RepC did not bind 73 bp PCR product including three tandem repeats (5'-CAACAAA- $\left.3^{\prime}\right)$, the binding sites for RepB and any other regions on pJB01. However, when RepB and RepC were added simultaneously in the reaction mixture for gel mobility shift assay, unexpectedly, three kinds of retarded bands were observed. It suggests that RepC can interact with RepB by protein-protein interaction. In addition, the copy numbers of RepC-deleted pJB01 ermC (erythromycin-resistant methylase C) plasmids are increased 1.37-1.45 folds when compared with that of parent pJB01 ermC. From these, it could be proposed that RepC plays a role as a negative regulator to modify Rep $B$ function in the initiation of pJB01 replication, and therefore, the copy number of pJB01 is maintained via mutual global regulation of various replication factors, such as CopA, ctRNA, RepB and RepC.

Key words: pJB01, repABC operon, replication initiator, RepC, global regulation.

\section{INTRODUCTION}

Genetic analysis of replication control mechanisms had first been attempted for plasmid R1 through isolation of mutants to increase copy number (Nordstrom et al.,

*Corresponding author. E-mail: jdbahk@gnu.ac.kr. Tel: 82-55751-6264. Fax: 82-55-759-9363.

Abbreviations: ctRNA, Counter-transcript ribonucleic acid; erm C, erythromycin-resistant methylase C; RT-PCR, reverse transcription polymerase chain reaction; RNA, ribonucleic acid; EDTA, ethylenediaminetetraacetic acid; DNA, deoxyribonucleic acid; TBE, Tris/Borate/ ethylenediaminetetraacetic acid; $\mathbf{N C B I}$, national center for biotechnology information; sso, single strand origin; dso, double strand origin; $\mathbf{R C}$, rolling-circle.
1972). The determinants of copy number control had been discovered in plasmid itself via characterization of these mutants, in which negative regulators (inhibitors) acting at the initiation step were involved in this control. A model of replication control by negative effectors was first proposed and described in quantitative terms by Pritchard et al. (1969). When a plasmid colonizes a new host, these negative regulators will be negligible owing to a little accumulated concentration of the inhibitors at initial stage. This seems desirable for successful establishment, since uninterrupted plasmid replication permit the normal copy number in a short time. Once the characteristic copy number is reached, maintenance of the average copy number in the population will then require adjustments to fluctuations in this value in individual cells. The control systems constantly maintain copy number by either 
increasing or decreasing the rate of replication per plasmid copy and cell cycle. Although mechanisms to counter-select newly replicated-plasmid molecules exist, for example, hemimethylation and supercoiling (Abeles et al., 1993; Nordstrom et al., 1984), individual plasmid copies are selected for replication at random from a pool including replicated and non replicated copies.

The inhibition of plasmid replication associated with an increase in the gene dosage of copy number control genes has been used to identify these genes (Pritchard, 1978; Nordstrom, 1985; Novick, 1987; Austin and Nordstrom, 1990; Kittell and Helinski, 1992; Chattoraj and Schneider, 1997). Control of replication by inhibitors requires measurement of the concentration of plasmid copies within the cell. This is probably achieved by an unstable inhibitor expressed constitutively or by a stable inhibitor synthesized shortly after each initiation event (Pritchard et al., 1969). The regulators via these alternatives modulate the initiation frequency after each initiation event and lead to increase or decrease in the rate of initiation of replication when the average copy number is, res-pectively, lower or higher than required. Otherwise, when the frequency of initiation is determined by the level of an initiator protein, one mechanism for controlled plasmid replication (unlike phage replication) includes inactivation of the initiator protein after each replication event (Rasooly and Novick, 1993; Wojtkowiak et al., 1993). In this study, in order to examine RepC function of pJB01, we performed RT-PCR (reverse transcription-PCR), gel mobility shift assay and constructs of repC-deleted mutants to demonstrate a single operon consisting of $\operatorname{cop} A$, repB and repC genes, protein-protein interaction between RepB and RepC, and copy number control of pJB01 by RepC, respectively.

\section{MATERIALS AND METHODS}

\section{Bacterial strains and plasmids}

A pathogenic Enterococcus faecium JS2 (Amps, Erms ${ }^{\mathrm{s}}$, Tet ${ }^{\mathrm{s}}$, and Van'), a strain non-harboring pJB01 plasmid, was isolated from patients in Samsung Biomedical Research Institute in Korea. In order to clone pJB01, Escherichia coli TG1 strain was employed. Cloned plasmids were transformed to $E$. faecium JS2 by the electroporation method (Dunny et al., 1991; Bensing and Dunny, 1993). Transformed plasmids were isolated from $E$. faeium JS2 by a modified alkaline lysis method (Sambrook et al., 1989; O'Sullivan and Klaenhammer, 1993). For selection of a host harboring target plasmids from E. coli TG1 and E. faecium JS2, an ermC (Khan et al., 2002) gene of plasmid pGKV21, after amplification by PCR using the portion of pE194 from this plasmid (van der Vossen et al., 1985), was transferred into pJB01 and named pJB01 ermC.

\section{RT-PCR (reverse transcription polymerase chain reaction) - RT- PCR}

Total RNAs were prepared from exponentially growing E. faecium JS2 cultures harboring pJB01 ermC (RNeasy Mini Kit, Qiagen). For the $1^{\text {st }}$ strand of three cDNAs containing $\operatorname{cop} A$ and a part of repB genes, $\operatorname{cop} A, \operatorname{rep} B$ and a part of repC genes, and $\operatorname{cop} A, \operatorname{Rep} B$ and RepC genes, each of oligonucleotides such as the $3^{\prime}$-end of RepB as 5'-AATTTCATAAAAGCTTCCCC-3', the 3'-end of E104Y as 5'GTCTTTTGAATAATGCGTTAAATAC-3' and the $3^{\prime}$-end of RepC as 5'-AAGCTTTAGTTATCCGCCCTTTCAAC-3' was annealed to total Ribonucleic acid (RNA) $(15 \mu \mathrm{g})$ at $65^{\circ} \mathrm{C}$ for $5 \mathrm{~min}$, and continuously, the reactions were done as described by a Superscript ${ }^{T M} \mathrm{RNaseH}^{-}$ RTase catalogue of Invitrogen. The next PCR was performed by the above three kinds of PCR products used as templates, and the $5^{\prime}$ end of CopA [5'-GGATCCATGGCTAGAGAAAAATCAGA-3'] as the $5^{\prime}$-end primer and the same oligonuclotides used for cDNA syntheses as the $3^{\prime}$-end primers.

\section{Purification of repC and repB and gel mobility shift assay}

Each of repB and repC genes was amplified by PCR and subcloned to the pQE30 vector for over expression. Subcloned repB and repC genes were over expressed by T7 promoter in E. coli JM109, and then, purified according to manufacturer's directions (Invitrogen, Korea). A 73-nucleotide stretch from nucleotides 583 to 655 , the three tandem repeats-containing region of plasmid pJB01, was amplified by PCR. One pmol of each PCR product was incubated with various concentrations of RepB and RepC in $20 \mu$ of reaction buffer [NB buffer $(20 \mathrm{mM}$ Tris. $\mathrm{HCl}, \mathrm{pH} \quad 8.0 ; 1 \mathrm{mM}$ Ethylenediaminetetraacetic acid (EDTA), pH 8.0; $100 \mathrm{mM} \mathrm{KCl} ; 5$ $\mathrm{mM}$ dithiothreitol), $50 \mu \mathrm{g} / \mathrm{ml}$ calf thymus Deoxyribonucleic acid (DNA) and $50 \mu \mathrm{g} / \mathrm{ml}$ bovine serum albumin] for $30 \mathrm{~min}$ at room temperature. Reactions were stopped by adding non-denaturing loading buffer [20\% (v/v) glycerol, 0.01\% (w/v) Bromophenol Blue in Tris/Borate/ Ethylenediaminetetraacetic acid (TBE) buffer]. Reaction products were separated on $5 \%$ native polyacrylamide gels. The resolved bands were stained with Ethidium Bromide $(0.5 \mu \mathrm{g} / \mathrm{ml})$ in TBE buffer for $30 \mathrm{~min}$, and destained with distilled water for 30 to $120 \mathrm{~min}$.

Site-directed mutagenesis of the pJB01 repC region and copynumber calculation

All mutants were obtained by inverse PCR (Mcpherson et al., 1993) using pJB01 erm $C$ as a template. To remove repC gene from the parent plasmid pJB01 ermC, the $3^{\prime}$-end of RepB as 5'AATTTCATAAAAGCTTCCCC-3' and the $5^{\prime}$-end of Em 5'GCTAGCATCGATTCACAA AAAATAGG-3' oligonucleotides were used as primers. The desired mutant plasmids were harvested by using E. coli TG1, and then, transformed to E. faecium JS2. To calculate their copy numbers, cultural broths of E. faecium JS2 harboring plasmids were grown to mid-exponential phase, and adjusted to 0.5 optical densities at $600 \mathrm{~nm}$ in wavelength. Equal aliquots of each culture were withdrawn. Cells were collected by centrifugation and plasmids were prepared by an Accuprep ${ }^{\mathrm{TM}}$ plasmid extraction kit (Bioneer, Korea). Isolated plasmids were separated on agarose gels and stained by Ethidium Bromide (0.5 $\mu \mathrm{g} / \mathrm{ml}$ ). Monomer covalently closed circular plasmids were quantified by Gel doc 2000 (Bio-Rad, USA) and covalently closed monomers of mutant plasmids were compared with that of parent pJB01 ermC.

\section{RESULTS AND DISCUSSION}

Demonstration of a single repABC operon and its surrounding structure

The plasmid pJB01 (GenBank accession number 
AY425961) was isolated originally from $E$. faecium JC2 (Kim et al., 2006), but it was not harbored by E. faecium JS2. It was assumed that this plasmid should have a single operon which consists of three orfs as $\operatorname{cop} A$, repB and $r e p C$ by the sequencing analysis using a Translation of ExPASy tool. This operon begins transcription at T695 or A696 on the pJB01 genetic map (Kim et al., 2008). CopA (as RepA in pMV158 and pE194)), the product of copA gene, binds to the promoter (or operator) region of the operon and regulates it at transcriptional level, as in case of pMV158 (del Solar et al., 1995, 1997) and pE194 (Kwak and Weisblum, 1994). RepB, as a product of repB gene and a replication initiator, performs nicking and nick/closing reactions on the nick site in the dso region (Kim et al., 2006). However, the corresponding function of RepC, the product of repC gene, has not been identified yet, through primary sequence analysis using the BLAST search of NCBI (National Center for Biotechnology Information). The transcription of $\operatorname{cop} A$ (171 nts), repB (666 nts), and repC (462 nts) genes begin at the $721^{\text {st }}$, $964^{\text {th }}$ and $1661^{\text {st }}$ nts and terminate at the $891^{\text {st }}, 1629^{\text {th }}$ and $2122^{\text {nd }} \mathrm{nts}$, respectively. Since consensus sequences of the promoter region were not existed in the intergenic regions of 72 nts (copA-repB) and/or 31 nts (repB-repC), it was thought that these genes are a single operon. As shown in Figure 1, RT- PCR results using each oligonucleotide set also revealed that these genes consist of a single operon.

\section{Binding on three tandem repeats by protein-protein interaction of RepB and RepC}

Following termination of the leading strand replication of pT181 plasmid, repC as an initiator protein is released with a short oligonucleotide attached to one subunit of homodimer repC, which prevents it from being recycled, as an essential feature of the plasmid's replication control system. It was reported that the oligonucleotide probably results from the passage of the replication fork past the RepC nick site after one round of replication, the subsequent cleavage and re-ligation of the DNA by repC (Khan, 1997; Zhao and Khan, 1997). Although repC can bind to the recognition sequence in the leading strand origin, repC*, as a modified one with a short oligonucleotide, cannot induce cruciform extrusion, which is an essential structure for replication initiation. RepC $^{*}$ is defective in its ability to oligomerize on the DNA for the next round replication. DNA binding and replication activities of RepC*$^{*}$ is greatly decreased, implying that it may play only a minor regulatory role in $\mathrm{pT} 181$ replication in vivo (Zhao et al., 1998).

On the contrary, Rep proteins of pMV158 family do not lead to a covalent binding with nicked DNA in itself (Moscoso et al., 1997). Therefore, it should be existing in other mechanism for deactivation of generated Reps in its family including pJB01. Since in this work the pJB01
repC-RepC gene was co-expressed as a component of the polycistronic mRNA of copA and repB gene, as shown in Figure 1, it could not be excluded from the possibility that RepC might play a role as a regulator of pJB01 replication through modification of RepB. In Figure 2, RepC did not show binding on the DNA segment containing three tandem repeats, known as DNA binding sites for RepB (as an initiator). However, simultaneous addition of RepB and RepC, particularly in higher concentrations of RepC, showed three extra retarded bands compared to the case of RepB only. It suggests that RepC interacts with RepB by protein-protein interaction. Interestingly, plasmid pLC2 (Vogel et al., 1991) which belongs to pMV158 family seems to be very similar to plasmid pJB01, in the aspect of the organization of sso (single strand origin), dso (double strand origin) and genes encoding for replication initiation factors. Particularly, unidentified pLC2 orf 3 , which composed of 153 amino acid residues and a weakly acidic protein as a theoretical pl of 5.11, is homologous up to $96 \%$ to corresponding pJB01 RepC (Figure 3). Based on the similar organization and amino acid sequence homology, it could not be excluded from the possibility that pLC2 orf 3 also regulates the initiation of replication via protein-protein interaction with orf 2 .

\section{Increase of the copy numbers of the repC-deleted mutants}

In order to examine the role of RepC in the regulation of pJB01 copy number, three mutants of RepC-deleted pJB01 ermC were created by inverse PCR (Figure 4a). All RepC-deleted pJB01 ermC mutants increased the copy numbers of 1.37-1.45 times when compared with that of parent plasmid pJB01 ermC (Figure 4b). In our previous work (Kim et al., 2008), the copy numbers of ctRNA mutants showed 1.78-5.43 times higher than that of parent pJB01 ermC. Based on these, it is assumed that RepC inhibits the initiation of replication weakly by modification of RepB, on the contrary to ctRNA does as a main repressor on the translation level.

\section{A hypothetical model for the copy number control of pJB01}

Based on all the data obtained in this work, RepC seems to be involved in the regulatory system of pJB01 DNA replication, in addition to various factors such as CopA, ctRNA and RepB. In some detail, according to our study it was demonstrated that CopA represses transcription of a repABC operon by interaction on partial palindromic sequence formed near the -35 box consensus sequence in the promoter region (unpublished data). In addition, pJB01 ctRNA, a 54 nucleotide transcript encoded on the opposite strand from the cop $A / r e p B$ intergenic region and 

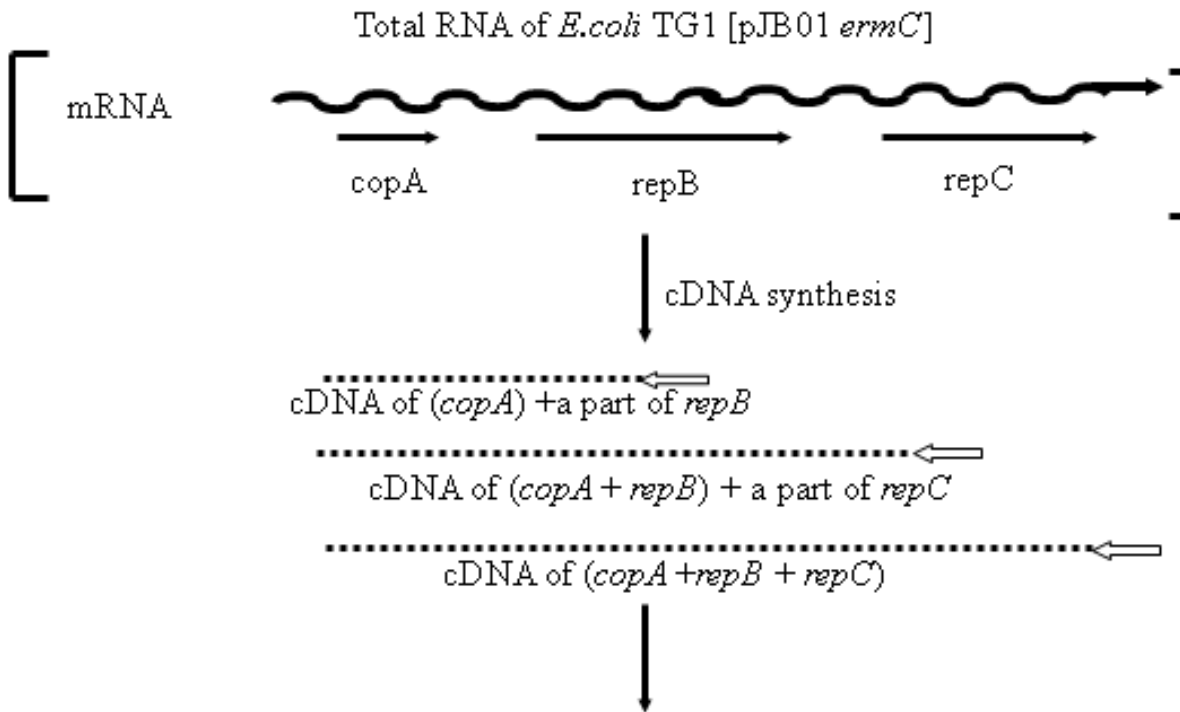

Amplification by PCR

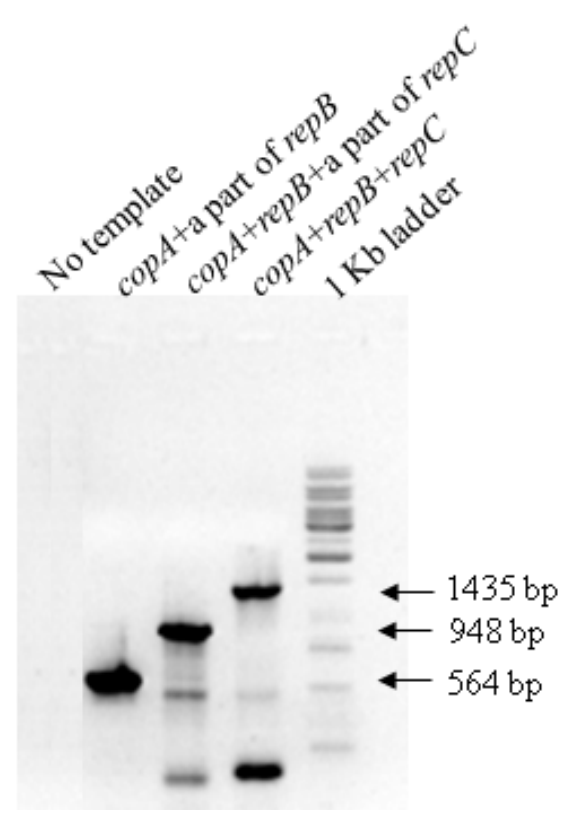

Figure 1. Demonstration of a single operon system consists of $\operatorname{cop} A$, repB and repC cistrons. Each primers marked by open arrows at the experimental strategy in the upper panel was annealed to total RNA isolated from E. coli TG1 culture harboring the pJB01 ermC, and extended by reverse transcriptase. The RT-PCR products consist of 564, 948 and 1435 bps as indicated on the right side of the gel, respectively. A full-length mRNA transcript of the $\operatorname{rep} A B C$ operon was represented by a thick-waved arrow. Each cistrons of a putative operon was marked below the repABC mRNA, primers for the first cDNA synthesis were represented in open arrows, and their reverse transcripts were shown in dotted lines. PCR products were separated on a $1 \%$ agarose gel and those products corresponding to three parts of a putative operon were marked by arrows on the right of the gel image.

also negatively regulates RepB expression on the translational level by partially overlapping an ARBS (Kim et al., 2008). Interestingly, RepB promotes replication activity on appropriate concentrations, but under overexpressed conditions, it not only decreases the replication activity of plasmid pJB01 but threatens viability of its host (unpublished data). Since the initiators of many rollingcircle $(\mathrm{RC})$ plasmids do rate-limiting for replication, these proteins seems to be usually inactivated after accomplishment of one round of replication. In the pT181 plasmid, inactivation of the initiator RepC protein occurs by the attachment of an oligonucleotide to its active 


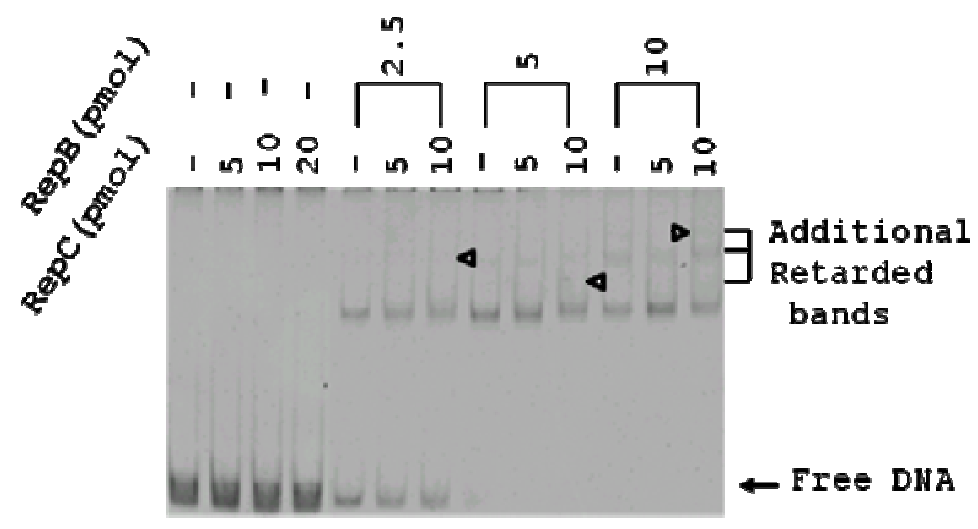

Figure 2. Confirmation of the protein-protein interaction between RepB and RepC by gel-shift assay. Free DNA indicates 73 bps PCR product containing three tandem repeats. Three additional retarded bands were produced by simultaneous addition of RepB and RepC to the reaction mixture for gel-shift assay. Concentrations of RepB and RepC added in pmol are indicated on the top of the gel image.



Figure 3. Comparison of replication factors between pJB01 and pLC2. (A) A schematic model of their gene organizations. Plasmid pJB01 is the same as pLC2 in view of gene organization, for example, orientations and arrangements of $d s o, s s o$, and three orfs. (B) Some features of their replication factors. Various features of replication factors in two plasmids were compared such as direct/tandem repeats, and orfs including origin of plasmids and prototype of RCR. (C) Alignment of the amino acid sequence of RepC in pJB01 with that in pLC2. Two RepCs show about $96 \%$ homology. Upper-case letters are residues conserved more than $90 \%$, Lower-case letters are residues conserved 50 to 90\%, and \# indicates the NDQE conserved positions.

tyrosine residue (Rasooly and Rasooly, 1996; Jin et al., 1996; Zhao et al., 1998). However, since Rep proteins as a replication initiator of pMV158 family do not bind covalently with an oligonucleotide after one round replication, its inactive forms are not observed (Moscoso et al., 1997), on the contrary to pT181 RepC.

Therefore, pMV158 family may need a new system for preventing accumulation of active Rep proteins, in other 
A

\begin{tabular}{|c|c|c|}
\hline Mutant name & Deleted region & Description \\
\hline $23-2$ & $\begin{array}{l}\text { pJB01A1667- } \\
2167 / G C T A G C(E m 5)\end{array}$ & $\begin{array}{l}\text { PCR primer : RepB } 3^{\prime} \text { end-Em } 5^{\prime} \text {, total } \\
\text { synthesized size }: 2746 \mathrm{bp}\end{array}$ \\
\hline $23-3$ & $\begin{array}{l}\text { pJB 01A1669- } \\
2167 / C T A G C\left(\mathrm{Em}^{\prime}\right)\end{array}$ & $\begin{array}{l}\text { Deletion of } G \text { residue in NheI site }\left(5^{\prime}-\right. \\
\left.\text { GCTAGC- } 3^{\prime}\right) \text {, total synthesized size : } \\
2747 \mathrm{bp}\end{array}$ \\
\hline $23-5$ & $\begin{array}{l}\text { pJB01A1669- } \\
2167 / G C T A G C(E m 5)\end{array}$ & total synthesized size : $2748 \mathrm{bp}$ \\
\hline
\end{tabular}

$\mathbf{B}$

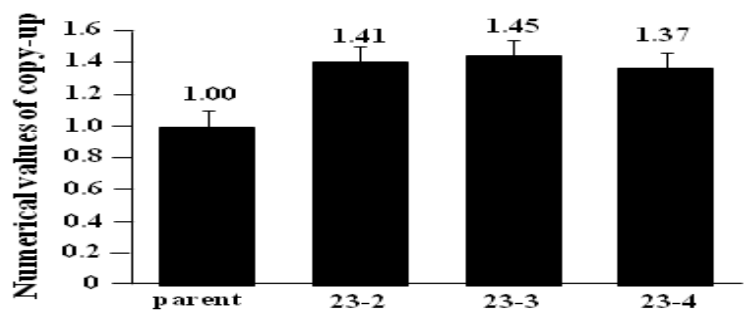

Names of pJBo 1 derivatives

Figure 4. Changes of copy numbers of a few pJB01 ermC $\Delta r e p C$ mutants. (A) Constructions of three pJB01 ermC $\triangle r e p C$ mutants. The mutants were constructed by deletion of most parts of the repC gene, remaining some amino acid residues encoded at start and end portions. (B) Copy number ratios of three repC mutants. X-axis represents names of pJB01 ermC and its repC mutants as 23-2, -3 and 4 , and $\mathrm{Y}$-axis indicates the copy-up ratios of three mutants against parent plasmid pJB01 ermC.

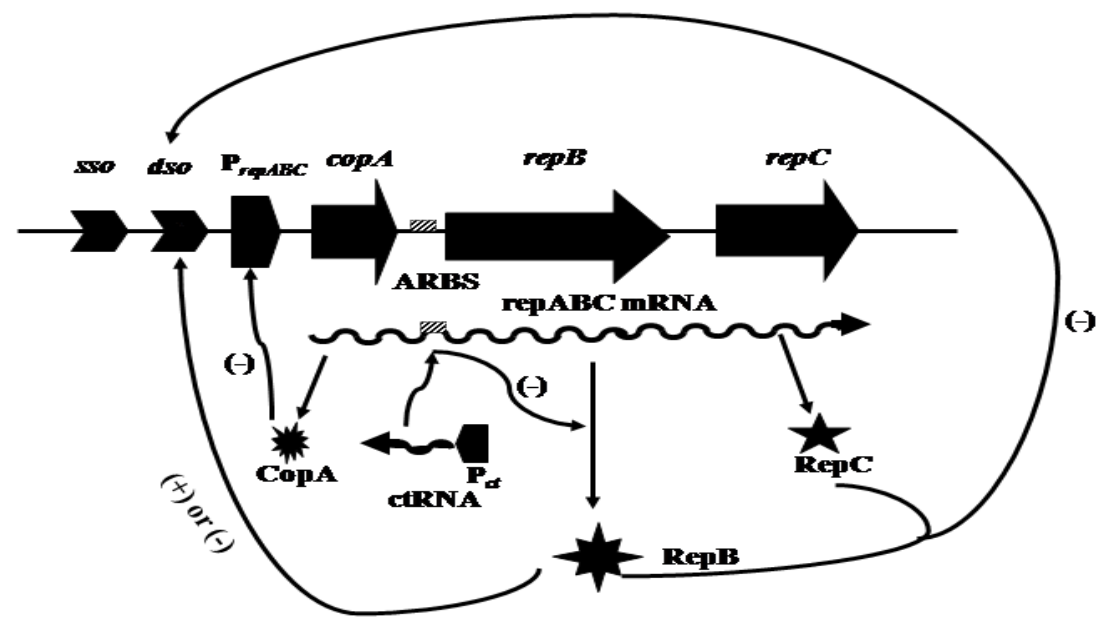

Figure 5. A hypothetical model for copy number control of pJB01. RepB is a positive regulator in the initiation of $\mathrm{pJB} 01 \mathrm{RC}$ replication at a normal level in vivo, whereas it functions as a negative regulator at an overexpressed level. The $\operatorname{cop} A, \operatorname{rep} B$ and repC genes consist of a single operon, repABC and its transcription is autoregulated through CopA's binding on the putative operator region $\left(\mathrm{P}_{\text {repABC }}\right)$. Furthermore, the translation of $r e p B$ is regulated by binding of ctRNA to the ARBS positioned between $\operatorname{cop} A$ and repB. On these points of view, including co-expression of $\operatorname{cop} A$ and rep $B$ with $r e p C$ as a polycistronic mRNA, it could be assumed that RepC plays negative roles in the initiation of plasmid pJB01 replication through RepB-RepC interaction.

words, for rate-limiting of replication. Based on our data in this work, it could be hypothesized that RepC modifies RepB by protein-protein interaction and this leads to lessen the optimal initiation activity of RepB replication. It is supported in that the repC-delteted mutants show a few copy-ups by $1.37-1.45$ times against parent pJB01 ermC plasmid (Figure 4). The copy number control of pJB01 might be hypothesized as shown in Figure 5 . The dso is 
controlled positively or negatively depending on the expressed amounts of RepB. CopA binds a putative operator and then, auto-regulates transcription of the repABC operon. The ctRNA inhibits translation of RepB by binding on ARBS. RepC interacts with RepB and may play a role as a putative negative regulator at the $d s o$. In this study, it was demonstrated that $\operatorname{cop} A, \operatorname{rep} B$ and repC genes consist of a single operon, and that RepC interacts with RepB by protein-protein interaction. Moreover, a few copy-ups of its repC-deleted mutants against parent pJB01 ermC plasmid were observed. Taken all, it was suggested that the maintenance of the coy number of pJB01 is accomplished by mutual interactions of various replication factors, such as CopA, ctRNA, RepB and RepC.

\section{ACKNOWLEDGEMENTS}

This work was supported by Priority Research Centers Program (2009-0093813) through the National Research Foundation of Korea (NRF) funded by the Ministry of Education, Science and Technology of Korea and by Technology Development Program for ('Agriculture and Forestry' or 'Food' or 'Fisheries'), Ministry for Food, Agriculture, Forestry and Fisheries and Republic of Korea.

\section{REFERENCES}

Abeles AL, Brendler T, Austin SJ (1993). Evidence of two levels of control of $\mathrm{P} 1$ oriR and host oriC replication origins by DNA adenine methylation. J. Bacteriol., 175: 7801-7807.

Austin SJ, Nordstrom K (1990). Partition-mediated incompatibility of bacterial plasmids. Cell, 60: 351-354.

Bensing BA, Dunny GM (1993). Cloning and molecular analysis of genes affecting expression of binding substance, the recipientencoded receptor(s) mediating mating aggregate formation in Enterococcus faecalis. J. Bacteriol., 175(22): 7421-7429.

Chattoraj DK, Schneider TD (1997). Replication control of plasmid P1 and its host chromosome: the common ground. Prog. Nucleic Acid Res. Mol. Biol., 57: 145-186.

del Solar G, Acebo P, Espinosa M (1995). Replication control of plasmid pLS1: efficient regulation of plasmid copy number is exerted by the combined action of two plasmid components, CopG and RNAll. Mol. Microbiol., 18(5): 913-924.

del Solar G, Acebo P, Espinosa M (1997). Replication control of plasmid pLS1: the antisense RNAll and the compact rnall region are involved in translational regulation of the initiator RepB synthesis. Mol. Microbiol., 23(1): 95-108.

Dunny GM, Lee LN, LeBlanc DJ (1991). Improved electroporation and cloning vector system for Gram-positive bacteria. Appl. Environ. Microbiol., 57(4): 1194-1201.

Jin R, Zhou X, Novick RP (1996). The inactive pT181 initiator heterodimer, RepC/C*, binds but fails to induce melting of the plasmid replication origin. J. Biol. Chem., 271(49): 31086-31091.

Khan SA (1997). Rolling-circle replication of bacterial plasmids. Microbiol. Mol. Biol. Rev., 61: 442-455.

Khan AA, Nawaz MS, Khan SA, Steele R (2002). Detection and characterization of erythromycin-resistant methylase genes in Grampositive bacteria isolated from poultry litter. Appl. Microbiol. Biotechnol., 59: 377-381.
Kim SW, Jeong EJ, Kang HS, Tak JI, Bang WY, Heo JB, Jeong JY, Yoon GM, Kang HY, Bahk JD (2006). Role of RepB in the replication of plasmid pJB01 isolated from Enterococcus faecium JC1. Plasmid. 55: 99-113.

Kim SW, Jeong IS, Jeong EJ, Tak JI, Lee JH, Eo SK, Kang HY, Bahk JD (2008). The terminal and internal hairpin loops of the ctRNA of plasmid pJB01 play critical roles in regulating copy number. Mol. Cells, 26(1): 26-33.

Kittell BL, Helinski DR (1992). Plasmid incompatibility and replication control, 223-242. In D. B. Clewell (ed.), Bacterial conjugation. Plenum Press, New York, N.Y.

Kwak JH, Weisblum B (1994). Regulation of plasmid pE194 replication: control of cop-repF operon transcription by Cop and of repF translation by countertranscript RNA. J. Bacteriol., 176(16): 50445051.

Mcpherson MJ, Quirke P, Taylor GR (1993). PCR, A practical Approach. IRL Press, pp. 207-209.

Moscoso M, Eritja R, Espinosa M (1997). Initiation of replication of plasmid pMV158: Mechanisms of DNA strand-transfer reactions mediated by the initiator RepB protein. J. Mol. Biol., 268: 840-856.

Nordstrom K (1985). Control of plasmid replication: theoretical considerations and practical solutions, p. 189-214. In D. R. Helinski, S. N. Cohen, D. B. Clewell, D. A. Jackson, and A. Hollaender (ed.), Plasmids in bacteria. Plenum Press, New York, N.Y.

Nordstrom K, Ingram LC, Lundback A (1972). Mutations in R factors of Escherichia coli causing an increased number of R-factor copies per chromosome. J. Bacteriol., 110: 562-569.

Nordstrom K, Molin S, Light J (1984). Control of replication of bacterial plasmids: genetics, molecular biology, and physiology of the plasmid R1 system. Plasmid., 12: 71-90.

Novick RP (1987). Plasmid incompatibility. Microbiol., Rev., 51: 381-395.

O'sullivan DJ, Klaenhammer TR (1993). Rapid mini-prep isolation of high-quality plasmid DNA from Lactococcus and Lactobacillus spp. Appl. Environ. Microbiol., 59: 2730-2733.

Pritchard RH (1978). Control of DNA replication in bacteria, p. 1-22. In Molineux, I. and Kohiyama, M. (ed.), DNA synthesis: present and future. Plenum Press, New York, N. Y.

Pritchard RH, Barth PT, Collins J (1969). Control of DNA synthesis in bacteria. Symp. Soc. Gen. Microbiol., 19: 263-297.

Rasooly A, Novick RP (1993). Replication-specific inactivation of the pT181 plasmid initiator protein. Science, 262: 1048-1050.

Rasooly A, Rasooly RS (1996). Modification of the plasmid initiator protein RepC active site during replication. FEMS Microbiol. Lett., 145: 245-253.

Sambrook J, Fritsch EF, Maniatis T (1989). Molecular Cloning, A laboratory manual. $2^{\text {nd }}$ edition, Cold Spring Harbor Laboratory. Cold Spring Harbor, NY, 1.25 - 28, 1. 38-41.

van der Vossen JM, Kok J, Venema G (1985). Construction of cloning, promoter-screening, and terminator-screening shuttle vectors for Bacillus subtilis and Streptococcus lactis. Appl. Environ. Microbiol., 50(2): 540-542.

Vogel RF, Lohmann M, Weller AN, Hugas M, Hammes WP (1991). Structural similarity and distribution of small cryptic plasmids of Lactobacillus curvatus and L. sake. FEMS Microbiol. Lett., 68(2): 183-190.

Wojtkowiak D, Georgopoulos C, Zylicz M (1993). Isolation and characterization of ClpX, a new ATP-dependent specificity component of the Clp protease of Escherichia coli. J. Biol. Chem., 268: 22609-22617.

Zhao AC, Ansari RA, Schmidt MC, Khan SA (1998). An oligonucleotide inhibits oligomerization of a rolling circle initiator protein at the pT181 origin of replication. J. Biol. Chem., 273(26): 16082-16089.

Zhao AC, Khan SA (1997). Sequence requirements for the termination of rolling-circle replication of plasmid pT181. Mol. Microbiol., 24: 535544. 